\title{
In Vitro Validation of Antiparasitic Activity of PLA-Nanoparticles of Sodium Diethyldithiocarbamate against Trypanosoma cruzi
}

\author{
Johny Wysllas de Freitas Oliveira ${ }^{1,2}$ D, Mariana Farias Alves da Silva ${ }^{3,4}$ (D), Igor Zumba Damasceno ${ }^{5}$, \\ Hugo Alexandre Oliveira Rocha ${ }^{2,6}$, Arnóbio Antônio da Silva Júnior ${ }^{3,4}$ and Marcelo Sousa Silva ${ }^{1,2,4,7, *(D)}$
}

Citation: de Freitas Oliveira, J.W.; da Silva, M.F.A.; Damasceno, I.Z.; Rocha, H.A.O.; da Silva Júnior, A.A.; Silva, M.S. In Vitro Validation of Antiparasitic Activity of PLA-Nanoparticles of Sodium Diethyldithiocarbamate against Trypanosoma cruzi. Pharmaceutics 2022, 14, 497. https://doi.org/10.3390/ pharmaceutics14030497

Academic Editor:

Maria Nowakowska

Received: 25 January 2022

Accepted: 17 February 2022

Published: 24 February 2022

Publisher's Note: MDPI stays neutral with regard to jurisdictional claims in published maps and institutional affiliations.

Copyright: (C) 2022 by the authors. Licensee MDPI, Basel, Switzerland. This article is an open access article distributed under the terms and conditions of the Creative Commons Attribution (CC BY) license (https:// creativecommons.org/licenses/by/ $4.0 /)$.
1 Immunoparasitology Laboratory, Department of Clinical and Toxicological Analysis, Centre of Health Sciences, Federal University of Rio Grande do Norte, Natal 59012-570, Brazil; johny3355@hotmail.com

2 Programa de Pós-Graduação em Bioquímica, Department of Biochemistry, Centro de Biociências, Universidade Federal do Rio Grande do Norte, Natal 59078-970, Brazil; hugo-alexandre@uol.com.br

3 Laboratory of Pharmaceutical Technology and Biotechnology, Department of Pharmacy, Federal University of Rio Grande do Norte-UFRN, Natal 59012-570, Brazil; marianafarias0011@gmail.com (M.F.A.d.S.); arnobiosilva@gmail.com (A.A.d.S.J.)

4 Programa de Pós-graduação em Ciências Farmacêuticas, Department of Pharmacy, Centro de Ciências da Saúde, Universidade Federal do Rio Grande do Norte, Natal 59012-570, Brazil

5 Departamento de Engenharia de Materiais, Department of Engineer, Centro de Tecnologia, Universidade Federal do Rio Grande do Norte, Natal 59078-970, Brazil; igorzumba@ufrn.edu.br

6 Laboratório de Biotecnologia de Polímeros Naturais-BIOPOL, Departament of Biochemistry, Centro de Biociências, Universidade Federal do Rio Grande do Norte, Natal 59078-970, Brazil

7 Global Health and Tropical Medicine, Instituto de Higiene e Medicina Tropical, Universidade Nova de Lisboa, 1349-008 Lisbon, Portugal

* Correspondence: mssilva@ihmt.unl.pt or mssilva.ufrn@gmail.com

Abstract: Trypanosoma cruzi is a protozoan parasite responsible for Chagas disease, which affects millions around the world and is not treatable in its chronic stage. Sodium diethyldithiocarbamate is a compound belonging to the carbamate class and, in a previous study, demonstrated high efficacy against T. cruzi, showing itself to be a promising compound for the treatment of Chagas disease. This study investigates the encapsulation of sodium diethyldithiocarbamate by poly-lactic acid in nanoparticles, a system of biodegradable nanoparticles that is capable of reducing the toxicity caused by free DETC against cells and maintaining the antiparasitic activity. The nanosystem PLA-DETC was fabricated using nanoprecipitation, and its physical characterization was measured via DLS, SEM, and AFM, demonstrating a small size around $168 \mathrm{~nm}$ and a zeta potential of around $-19 \mathrm{mv}$. Furthermore, the toxicity was determined by MTT reduction against three cell lines (VERO, 3T3, and RAW), and when compared to free DETC, we observed a reduction in cell mortality, demonstrating the importance of DETC nanoencapsulation. In addition, the nanoparticles were stained with FITC and put in contact with cells for $24 \mathrm{~h}$, followed by confirmation of whether the nanosystem was inside the cells. Lastly, the antiparasitic activity against different strains of T. cruzi in trypomastigote forms was determined by resazurin reduction and ROS production, which demonstrated high efficacy towards T. cruzi equal to that of free DETC.

Keywords: sodium diethyldithiocarbamate; Trypanosoma cruzi; Chagas disease; nanoparticle; nanoprecipitation

\section{Introduction}

Chagas disease (CD) is a zoonosis caused by the flagellated parasite Trypanosoma cruzi that affects principally South America. CD has a spread of dispersion in many countries worldwide due to the immigration process in the risk zones carrying the disease, and this results in growing damage to non-endemic countries [1,2]. The pharmacological treatment 
for CD offered by the World Health Organization is benznidazole and nifurtimox. However, these drugs present high cytotoxicity, cause severe damage to the patients, and present low efficacy against chronic stages of CD due to their low bioavailability to reach the parasite inside the cells [3-5].

T. cruzi, the etiological agent of $C D$, has a complex life cycle and genomic diversity that allows it to survive and escape the immune system. Moreover, there is no efficient drug to treat the chronic stage of the disease. In previous studies, sodium diethyldithiocarbamate (DETC) demonstrated high antiparasitic activity against parasites Trypanosoma cruzi and Leishmania sp., which belong to the Trypanosomatidae family. The mechanism associated with its efficacy is based on the metal chelator activity and the stimulation of reactive oxygen species (ROS), causing damage to the parasite. The mechanisms are important to eliminate the parasite; however, they could cause some damage to the patient, as demonstrated in in vitro experiments [6-9].

One alternative to avoid the damage caused by DETC is using new technologies, and a promising alternative is the use of polymeric nanoparticles. Several studies already demonstrate the importance and benefits of nanosystems when applied to the treatment of parasitic diseases, reducing the cellular damage caused by some compounds, increasing the efficacy of drugs, and improving their bioavailability [10-13].

Poly-lactic acid (PLA), a biopolymer, is an aliphatic polyester approved by the Food and Drug Administration (FDA) for use as a biomaterial for therapeutic use. The PLA has important characteristics that have been demonstrated in the literature that make it useful as a polymer in polymeric nanoparticles. Several studies demonstrate its capacity to reduce cellular toxicity, increase permeability for compounds to enter in cells, and improve bioavailability [14-19]. These characteristics are important for the development of new treatments against T. cruzi in order to reduce the damage caused by new drugs and maintain their efficacy.

This work aims to associate the benefits of PLA nanoparticle systems demonstrated in the literature when coupled with the compound sodium diethyldithiocarbamate, which demonstrated, on a free stage, high efficacy against T. cruzi. Moreover, this association aims to reduce any damage that DETC can cause to cells, improve its bioavailability, and maintain the compound efficacy.

\section{Materials and Methods}

\subsection{Cells and Parasites Maintenance}

The cellular lineages RAW (ATCC number TIB-71), derived from macrophages, 3T3 (ATCC CRL-1658), derived from fibroblasts, and VERO (ATCC CCL-81), derived from epithelial kidney cells, were generously donated by the Biopolymers Laboratory (Natal, RN, Brazil) and were grown in DMEM medium (Cultilab, Campinas, SP, Brazil) supplemented with fetal bovine serum (FBS) at 10\% (v/v) (Cultilab, Campinas, SP, Brazil) and $100 \mathrm{U} / \mathrm{mL}$ penicillin and $100 \mu \mathrm{g} / \mathrm{mL}$ streptomycin. The cells were incubated at $37^{\circ} \mathrm{C}$ with $5 \% \mathrm{CO}_{2}$, and the medium was changed every three days for maintenance. The cells were further subculture at $80 \%$ confluence using a cell scraper (RAW cells) or trypsin/EDTA (3T3 and VERO cells).

Different strains of T. cruzi TcI (strain Dm28c and Bolivia) and TcII (strain Y) were cultured in an LIT (Liver Infusion Tryptose) medium supplemented with fetal bovine serum $(\mathrm{FBS})$ at $10 \%(v / v)$ and $100 \mathrm{UI} / \mathrm{mL}$ of streptomycin/penicillin. All strains were generously donated by the Laboratório de Parasitologia (FCFAr), Araraquara, Brazil. The culture of epimastigote forms of $T$. cruzi was maintained at $27^{\circ} \mathrm{C}$ in a BOD (incubator chamber, ASP, SP-500).

To obtain trypomastigote forms of different strains of T. cruzi, we utilized the method described by Contreras et al. [20]. Briefly, the parasites in medium LIT, after 5 to 7 days, achieved the stationary phase; then, the parasites were washed with PBS $1 \times \mathrm{pH} 7.4$ three times and resuspended in TAU medium. After $2 \mathrm{~h}$, we added $10 \mu \mathrm{M}$ of proline and sodium bicarbonate $1.4 \mathrm{M}$ in the proportion of 1:4 $(v / v)$. Then, the culture was kept for 
four days without movement at $27^{\circ} \mathrm{C}$ in a BOD. The metacyclogenesis was confirmed by optical microscopy (Biolab Brasil, São Paulo, Brazil).

\subsection{Preparation of PLA Nanoparticles of DETC Using Nanoprecipitation}

The poly-lactic acid (PLA) nanoparticles were prepared using nanoprecipitation with solvent evaporation methodology. The organic phase was composed of acetone PA (dielectric constant $\varepsilon$ 20.6) and ethanol PA ( $\varepsilon$ 24.6) in a volumetric ratio of 80:20. We used PLA at $0.5 \%(w / v)$ as a polymeric matrix, and sodium diethyldithiocarbamate (DETC) as the drug of choice to be incorporated into the system. The aqueous phase used polyvinyl alcohol (PVA) as a surfactant with a molar mass of $61.000 \mathrm{~g} / \mathrm{mol}$ and a viscosity of 9-11 mPa.s at a concentration of $0.25 \%(w / v)$. A volume of $6 \mathrm{~mL}$ of the organic phase was injected into $14 \mathrm{~mL}$ of the aqueous phase, maintaining the volumetric proportion ratio of 30:70 in a continuous flow of $1 \mathrm{~mL} / 29 \mathrm{~s}$. The phases were previously filtered through a nylon membrane of size $0.22 \mu \mathrm{m}$ and a cellulose acetate membrane of size $0.45 \mu \mathrm{m}$, respectively. Obtaining the nanoparticles was possible by completely removing the organic solvents under magnetic stirring at $720 \mathrm{rpm}$ at $25^{\circ} \mathrm{C}$, overnight.

\subsection{Physical and Chemical Characterization of DETC Nanoparticles}

The nanoparticles containing DETC were prepared using the solubilization in the organic phase component of ethyl alcohol (EtOH). DETC was added in 1:12 drug/polymer proportions, which corresponds to $8.2 \%(w / w)$ of the drug in the system. After adding DETC, the experiment followed the same procedure for obtaining nanoparticles without the drug, mentioned in the former section.

\subsubsection{Determination of Particle Diameter, Zeta Potential, and $\mathrm{pH}$}

In order to characterize the systems obtained, the particle diameter was determined using the light scattering methodology (Dynamic Light Scattering) in a ZetaSizer NanoZS analyser model (82 Brookhaven, Malvern, UK), at angle of detection of $173^{\circ}$. The measurement of the zeta potential was performed with the same equipment through electrophoretic mobility. Undiluted samples were analyzed using the pH meter (Gehaka, PG 1800, São Paulo, Brazil. All analyses were performed in triplicate at room temperature $25 \pm$ and data are expressed as mean \pm standard deviation (SD).

\subsubsection{Encapsulation Efficiency and Drug Loading}

To determine the encapsulation efficacy and drug loading, aliquots of $1 \mathrm{~mL}$ of the nanoparticle suspension samples with and without DETC were prepared. Then, the samples were centrifuged in an ultrafiltration device (Sartorius ${ }^{\circledR}$, Vivaspin 2, Ultra-15MWCO $10 \mathrm{kDa}$ ) (Eppendorf, Hamburg, Germany) using an Eppendorf ${ }^{\odot}$ 5804R refrigerated centrifuge (Sartorius AG, Argentina, Germany) $16.9 \mathrm{RCF}(\mathrm{g})$ for $60 \mathrm{~min}$ at $4{ }^{\circ} \mathrm{C}$. After obtaining the filtrate, the NP were analyzed via UV-Vis spectrophotometry (Thermo Fisher, Whalthan, MA, USA) using a spectrophotometer and a UV-Thermo Fisher Scientific evolution 60S spectrophotometer (Thermo Fisher, Whalthan, MA, USA). With the aid of the linear regression equation extracted from the standard curve built under the same conditions of analysis, the content of the drug incorporated in the systems was determined. Encapsulation efficiency (EE) and drug loading (DL) were calculated using Equations (1) and (2).

$$
\begin{gathered}
\mathrm{EE}(\%)=\frac{(\text { totaldrug }- \text { drugsupernatant })}{\text { totaldrug }} \times 100 \\
\mathrm{DL}(\%)=\frac{(\text { totaldrug }- \text { nanoparticles })}{\text { totalnanoparticles }} \times 100
\end{gathered}
$$

\subsubsection{Infrared Absorption Spectroscopy (FTIR-ATR)}

Spectroscopic analyses in the infrared region were carried out to investigate the drug interaction level with the structural components of the systems or the possible components' 
chemical changes. For this, the absorption spectra in the infrared region of the isolated structural components, the different formulations, and the respective physical mixtures of the same composition were obtained. The equipment used was a Transformed Fourier Infrared (FTIR)—ATR, SHIMADZU IR Prestige 21 (Shimadzu, Tokyo, Japan). The spectra were recorded with 20 scans at a resolution of $4 \mathrm{~cm}^{-1}$ wavenumbers between 4000 and $500 \mathrm{~cm}^{-1}$.

\subsubsection{Atomic Force Microscopy (AFM)}

The nanoparticles shape and size were observed using AFM images. The dispersions were previously dried in a desiccator for $24 \mathrm{~h}$. The measurements were performed using AFM (SPM-9700, Shimadzu, Kloten, Switzerland) at $25^{\circ} \mathrm{C}$ in a non-contact cantilever, with digitalization at $1 \mathrm{~Hz}$.

\subsubsection{Scanning Electron Microscopy (SEM)}

Samples analyzed using field emission scanning electron microscopy (SEM) (Model augira, Brand Carl Zeiss, Oberkochen, WB, GER) were fixed on a conductive adhesive surface and covered with approximately $20 \mathrm{~nm}$ gold in a BAL-TEC sputter coater (Bal-Tec, Scotia, USA), model SCD 005, in order to make them conductive. Analyses were performed with $25 \mathrm{kV}$ of electron acceleration voltage, using images formed by the secondary electron detector (ETD) (Model augira, Brand Carl Zeiss, Oberkochen, WB, GER).

\subsection{In Vitro DETC Release Study}

The in vitro release assay was performed on Franz diffusion cells. The dissolution medium employed was a $50 \mathrm{mM}$ monobasic potassium phosphate-buffered solution $(\mathrm{pH}=7.4)$. The systems remained in constant agitation at $360 \mathrm{rpm}$ and $37 \pm 2{ }^{\circ} \mathrm{C}$ during the whole experiment. At predetermined time intervals $(30,60,90,120,180,300$, and $360 \mathrm{~min}$ ), samples of aliquots of the release medium were collected through the sidearm of the Franz cell. The same volume of freshly buffered solution replaced the medium to maintain the sink conditions. Through the linear regression equation of the standard drug curve, the drug released from the nanoparticles was determined using UV-Vis spectrophotometry. The drug solution was prepared at concentrations equivalent to those present in the nanoparticles $(200 \mu \mathrm{g} / \mathrm{mL})$.

\subsection{Toxicity against Cellular Lineages}

To evaluate if the nanoparticles could cause toxicity, we performed MTT assay against three cellular lineages: RAW (ATCC number TIB-71), derived from macrophages, 3T3 (ATCC CRL-1658), derived from fibroblasts, and VERO (ATCC CCL-81), derived from epithelial kidney cells. The assay was performed according to the method described by Mosmman [21]. Briefly, the cells were plated in a 96-well plate in concentration of $5 \times 10^{3}$ cells/well and grown for $24 \mathrm{~h}$ in culture conditions. Hereafter, different concentrations of nanoparticle systems containing DETC (22 to $132 \mu \mathrm{M})$ and nanoparticle systems without DETC (22 to $132 \mu \mathrm{M}$ ) were applied in a 96-well plate and kept in culture conditions for $24 \mathrm{~h}$. After $24 \mathrm{~h}$, the culture medium was withdrawn, and to it was added $100 \mu \mathrm{L}$ of MTT at a concentration of ( $1 \mathrm{mg} / \mathrm{mL}$ dissolved in DMEM medium without FSB). Then, the cells were incubated for $4 \mathrm{~h}$ at $37{ }^{\circ} \mathrm{C}$ and $5 \% \mathrm{CO}_{2}$. Posteriorly, the culture supernatant was discarded, and to it was added $100 \mu \mathrm{L} /$ well of ethanol to solubilize the formazan crystals. The absorbance was measured with the equipment Epoch microplate spectrophotometer (Biotek Instruments Inc., Winooski, VT, USA) at $\lambda=570 \mathrm{~nm}$. Cell viability was calculated in relation to the negative control using the formula: \% viability $=($ Atest $/$ A Control $) \times 100$, in which Atest corresponds to the absorbance of the experimental group, and A Control corresponds to the absorbance of the negative control. 


\subsection{Fluorescence Nanoparticle Production and Capacity to Penetrate Cells}

To evaluate if the nanoparticles carrying DETC can penetrate cells, we produced fluorescent nanoparticles to which the cells were exposed. Briefly, the nanoparticles were lyophilized; $30 \mathrm{mg}$ of lyophilized nanoparticles were added to $1 \mathrm{mg}$ of fluorescein and dissolved in $10 \mathrm{~mL}$ of PBS $1 \times \mathrm{pH} 7.4$ under agitation for $1 \mathrm{~h}$. After this period, the excess of fluorescein was removed by dialysis using membranes of $6 \mathrm{kDA}$ for $4 \mathrm{~h}$ under agitation, and during this period the water was changed 3 times to facilitate dialysis. After dialysis, the nanoparticles were lyophilized again for the next steps.

To analyze if stained nanoparticles can penetrate the cells, we utilized the cellular lineages RAW and VERO. The cells were plated in glasses inside 24-well plates at a concentration of $3 \times 10^{4}$ cells/plate and kept for $24 \mathrm{~h}$ in DMEM medium supplemented with SFB at $37{ }^{\circ} \mathrm{C}$ and $5 \%$ of $\mathrm{CO}_{2}$. Hereafter, the stained nanoparticles were added for different periods (15 $\mathrm{min}, 30 \mathrm{~min}, 1 \mathrm{~h}, 2 \mathrm{~h}, 12 \mathrm{~h}$, and $24 \mathrm{~h}$ ) to evaluate if the time was an important factor when analyzing if the particles can penetrate the cell membrane. After this, the glasses containing the cells were fixed with methanol for $20 \mathrm{~min}$, removed, and washed 3 times in cold PBS $1 \times \mathrm{pH}$ 7.4. The images were obtained from different fields, randomly selected, which were analyzed using the NIS Elements AR v. 4.00.03 64-bit software (Nikon (2011), Melville, NY, USA) in blue spectra.

\subsection{In Vitro Antiparasitic Activity against Different Strains of Trypanosoma cruzi}

Trypomastigote forms of different strains of T. cruzi were used to perform this assay. Here, the parasites were plated in 96-well plates at a concentration of $1 \times 10^{7}$ parasites $/ \mathrm{mL}$ and exposed to different concentrations of DETC nanoparticles $(11 \mu \mathrm{M}$ at $132 \mu \mathrm{M})$ for $24 \mathrm{~h}$ at $28^{\circ} \mathrm{C}$. After exposure, the parasites were submitted to a colorimetric viability assay using resazurin, as previously used by the author of [9]. The results were plotted as a percentage $(\%)$ of parasite deaths. The selective index was calculated based on results of the $\mathrm{IC}_{50}$ of cell lines exposed to DETC and the $\mathrm{IC}_{50}$ of DETC against trypomastigote forms of T. cruzi. Benznidazole was used as a positive control in the antiparasitic test and the $\mathrm{IC}_{50}$ was measured too.

\subsection{Induction of ROS Production by Parasites Exposed to DETC Nanoparticles}

All strains of T. cruzi had their reactive oxygen species (ROS) production after treatment with nanoparticles of DETC detected using the marker $2^{\prime}, 7^{\prime}$-dichlorofluorescin diacetate (Sigma, Saint Louis, MO, USA). Briefly, the different strains of T. cruzi on epimastigote forms were treated with DETC nanoparticles at a concentration of $44.00 \mu \mathrm{M}$ for $24 \mathrm{~h}$ in the same conditions described previously. After treatment, the parasites were centrifuged at $2000 \mathrm{rpm}$ for $6 \mathrm{~min}$ at $4^{\circ} \mathrm{C}$ and washed with PBS pH 7.4 twice. Afterward, these parasites were loaded with $10 \mu \mathrm{M}$ of $2^{\prime}, 7^{\prime}$-dichlorofluorescin diacetate and maintained in a dark room for $45 \mathrm{~min}$. The endogenous ROS hydrogen peroxide $\left(\mathrm{H}_{2} \mathrm{O}_{2}\right)$ was used as a positive control of $0.5 \mathrm{mM}$. The ROS production was determined by the increase in fluorescence caused by the conversion of the probe and read with the equipment GloMax ${ }^{\circledR}$ Discover Microplate Read model GM 3000 (GloMax, Fitchburg, WI, USA) at $\lambda$ ex $=490-530 \mathrm{~nm}$.

\subsection{Statistical Analysis}

All experiments were executed in triplicate and as independent systems. The data were processed, and the results are presented in the form of mean \pm standard deviation. The data were submitted to the normality test of Shapiro-Wilk. The parametric results for 3 or more groups using the ANOVA test were further processed with Tukey's post hoc test, and for 2 groups with Student's $t$ test. Tests were performed using the software GraphPad Prism v. 7.0 (2016, GraphPad, San Diego, CA, USA) and P.A.S.T v. 2.17 (2012, PAST, Oslo, Norway). 


\section{Results}

\subsection{Physical and Chemical Properties of Nanoparticles of DETC via Nanoprecipitation}

DETC was incorporated at a 1:12 ratio of drug/polymer using a concentration of $0.5 \%$ PLA and $0.25 \%$ PVA to obtain NPD nanoparticles via the nanoprecipitation method (Figure 1A). The physicochemical properties of different drug-free (NPB) and drug-loaded (NPD) nanoparticles are shown in Table 1. The formation of a single particle family could be observed in the nanoparticle diameter distribution profile according to the light scattering intensity (Figure 1B). The quantitative analysis revealed that NPD resulted in an entrapment efficiency of around $72 \%$ and a drug loading level of $3.63 \%$ that corresponds to a final drug concentration of $200 \mu \mathrm{g} / \mathrm{mL}$. The structure observed in the results presented in Figure 1C,D represents the structure of NPB and NPD. It is possible to observe a uniform size of the nanoparticle structure and a low dispersion with regard to their conformation in both the AFM and SEM images.

A)

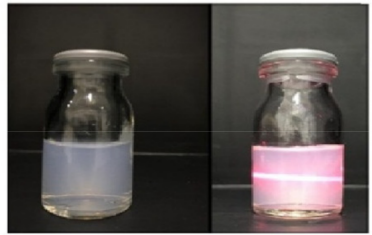

C)
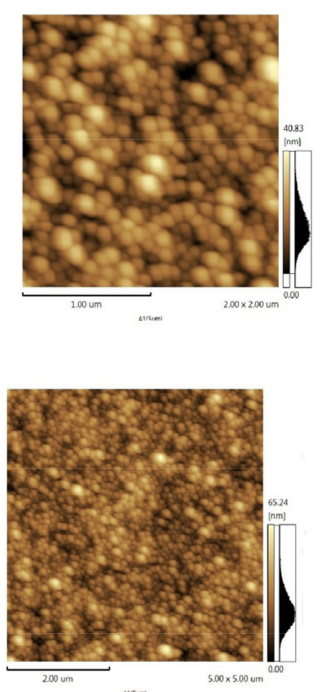

B)

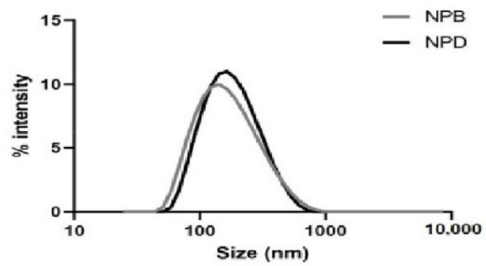

D)
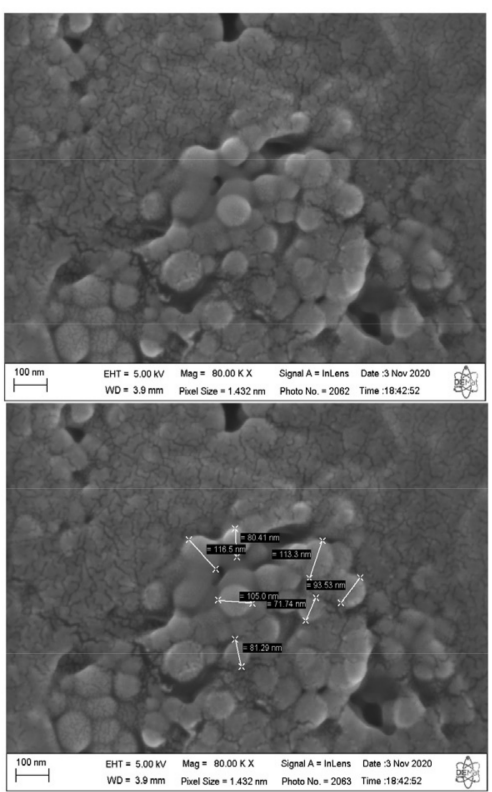

Figure 1. DETC-loaded nanoparticles. (A) The red-light scattering highlights the "Tyndall effect". (B) Particle size intensity distribution graph of DETC-loaded nanoparticles and drug-free nanoparticles. (C) Atomic force microscopy analysis of drug-free and DETC-loaded nanoparticles. (D) Scanning electron microscopy analysis of drug-free and DETC-loaded nanoparticles.

Table 1. Physically linked properties of the blank (NPB) and DETC-loaded nanoparticles (NPD). Results presented as mean \pm standard deviation in an independent triplicate system.

\begin{tabular}{ccccccc}
\hline Sample & Size $(\mathbf{n m}) \pm \mathbf{S D}$ & PdI $(\mathbf{n m}) \pm \mathbf{S P}$ & $\mathbf{Z P}(\mathbf{m V}) \pm \mathbf{S P}$ & $\mathbf{p H}$ & EE (\%) & DL (\%) \\
\hline $\mathrm{NPB}$ & $143.8 \pm 2.73$ & $0.154 \pm 0.08$ & $-21.80 \pm 2.75$ & 4.10 & - & - \\
NPD & $164.7 \pm 2.96$ & $0.221 \pm 0.02$ & $-19.50 \pm 5.15$ & 7.9 & 72.65 & 3.63
\end{tabular}

Note: SD (standard deviation); PdI (polydispersity index); ZP (zeta potential); EE (encapsulation efficiency); DL (drug loading). 


\subsection{Infrared Absorption Spectroscopy (FTIR-ATR)}

The FTIR spectra recorded for pure compost PLA and DETC, the physical mixture (PM) of PLA+DETC, and the nanosystems NPB and NPD are shown in Figure 2. In the PLA spectrum, it is possible to observe the presence of the stretching of the $\mathrm{C}=\mathrm{O}$ bond of the carbonyl groups at $1747 \mathrm{~cm}^{-1}$ and the $-\mathrm{CH}$ and $-\mathrm{CH}_{3}$ bands at $2995 \mathrm{~cm}^{-1}$ and $2945 \mathrm{~cm}^{-1}$ [22]. In the DETC spectra, between 2850 and $3000 \mathrm{~cm}^{-1}, \mathrm{C}-\mathrm{H}$ stretches are evident. The peak at $1415 \mathrm{~cm}-1$ demonstrated the absorption for group N-CS2. In 1128 and $2063 \mathrm{~cm}^{-1}$, we can observe the stretching $C=S$ and, between 600 and $1100 \mathrm{~cm}^{-1}$, we can observe the stretching $\mathrm{C}-\mathrm{S}, \mathrm{C}-\mathrm{C}, \mathrm{C}-\mathrm{N}, \mathrm{CH}_{2}$, and $\mathrm{CH}_{3}$. A region below $600 \mathrm{~cm}^{-1}$ corresponds to altered torsions [23-25]. The physical mixture presented important differences compared with NPD, mainly the intensity of the bands and the bathochromic shift of the $\mathrm{C}=\mathrm{O}$ bond of the carbonyl of ester band and the overlapping by DETC bands that occurred in the physical mixture. Furthermore, in PM, which resulted from a mixture of 1:1 (w/w) of PLA and DETC, it is possible to observe a reduction in the intensity of the DETC peaks when compared with free DETC and PLA peaks, which are not clearly visualized; however, the reduction in the DETC peaks indicates the presence of another compound.

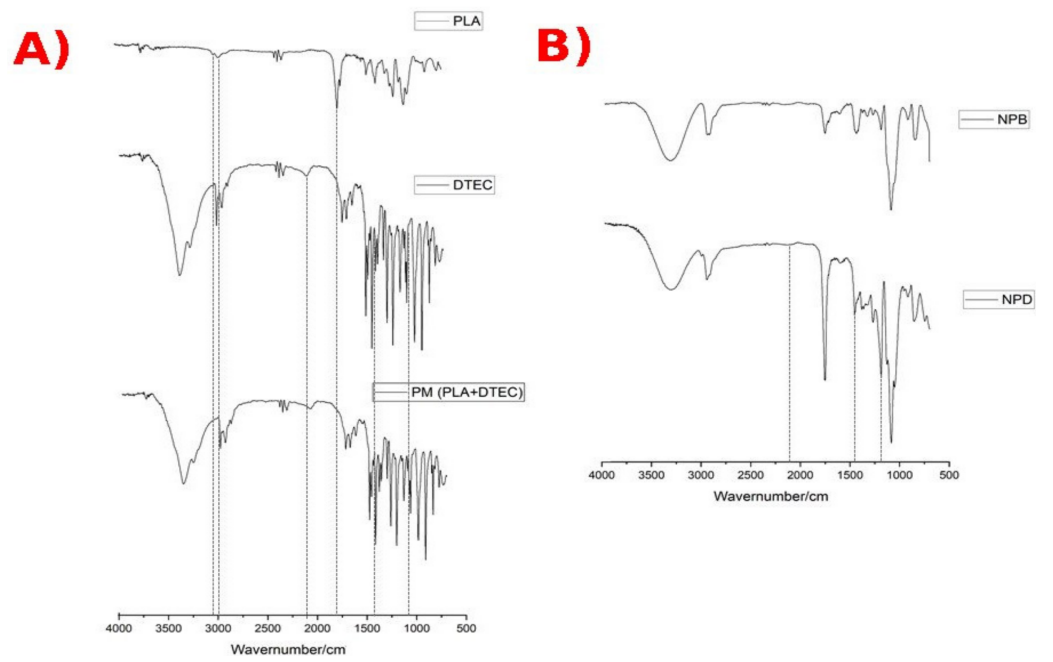

Figure 2. FTIR-ATR analysis. (A) Spectrum in region IV of the individual pure compounds and the compound physical mixture. (B) Spectrum in region IV of the drug-free (NPB) and DETC-loaded nanoparticles (NPD).

\subsection{In Vitro Drug Release}

The in vitro drug release assay is fundamental for understanding the physicochemical properties and mechanisms that influence drug release. Figure 3A shows the DETC release behavior from the DETC solution (DS) and from NPD. At $360 \mathrm{~min}$, DS showed a drug release of $48.3 \%$, while NPD showed a release of $15.6 \%$ at the same time. It was observed that the release of DETC from the nanoparticles was the slowest, representing a more controlled release than the drug solution. To determine the DETC release mechanism in NPD, mathematical kinetic models of the zero-order, first-order, Bhaskar, Freundlich, and parabolic types were applied [26-28]. After an analysis of the release determination coefficients, the parabolic diffusion model $\left(\left(\mathrm{M}_{\mathrm{t}} / \mathrm{M}_{\infty}\right) / \mathrm{t}=\mathrm{kt}^{-0.5}\right)$ showed the best fit $\left(\mathrm{R}^{2}>0.99\right)$ (Figure 3B).

\subsection{Cellular Toxicity of DETC Nanoparticles}

Three cell lines were used in the MTT assay to analyze the cytotoxicity of DETC nanoparticles. In Figure 4, we observed that RAW, 3T3, and VERO present a consistent curve when tested against different concentrations of nanoparticles. In Figure $4 \mathrm{~A}$, just the high concentration of $132 \mu \mathrm{M}$ of DETC nanoparticles caused a significant reduction in the cellular viability of VERO cells; on the other cell lines, we observed a constant result in 
cellular viability. Furthermore, we performed the test against the nanoparticles without DETC (Figure 4B), and possibly observed a similar profile to the DETC nanoparticles, except in the high concentration of $132 \mu \mathrm{M}$ against the VERO cells, where the viability was reduced to $60 \%$. During all experiments, DETC nanoparticles and nanoparticles without DETC did not cause high lethality to cell lines.

A)

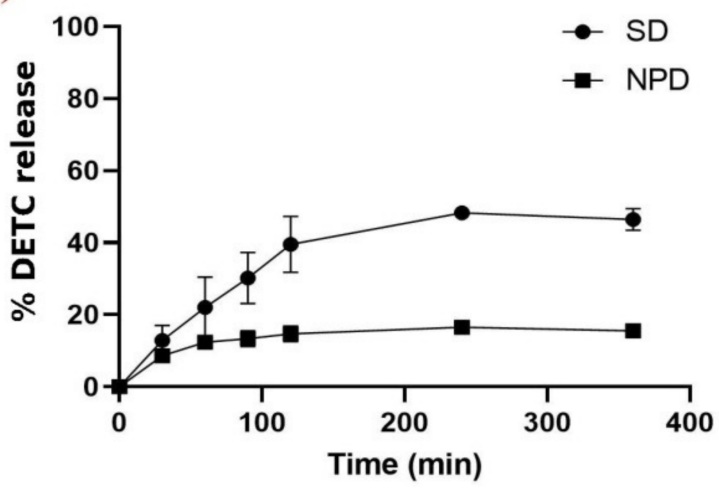

B)

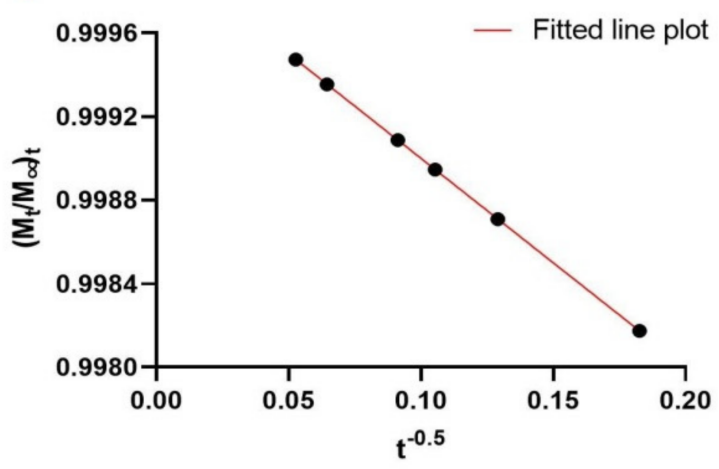

Figure 3. Experimental in vitro release profile. (A) Analysis of DETC solution (DS) and DETC-loaded nanoparticles (NPD) at the same time intervals and conditions. Results presented as mean \pm standard deviation in an independent triplicate system. (B) Parabolic model of NPD during performed assays. The dots are the points obtained by equation to determinate the R score.

A)

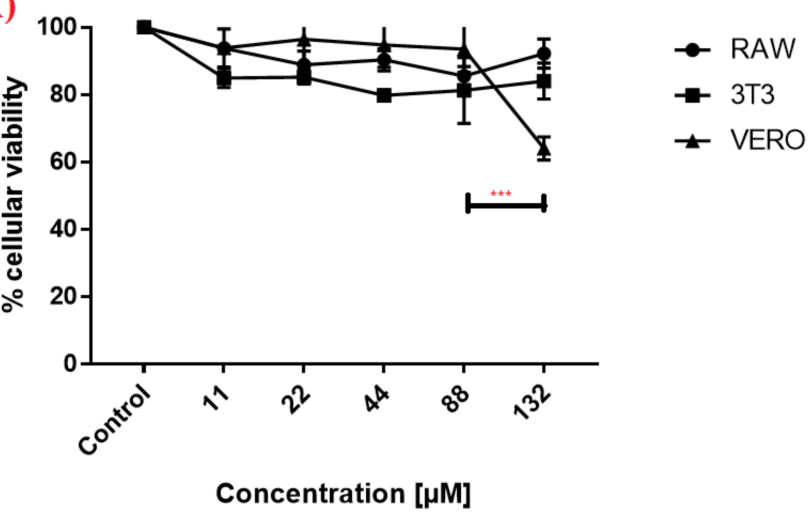

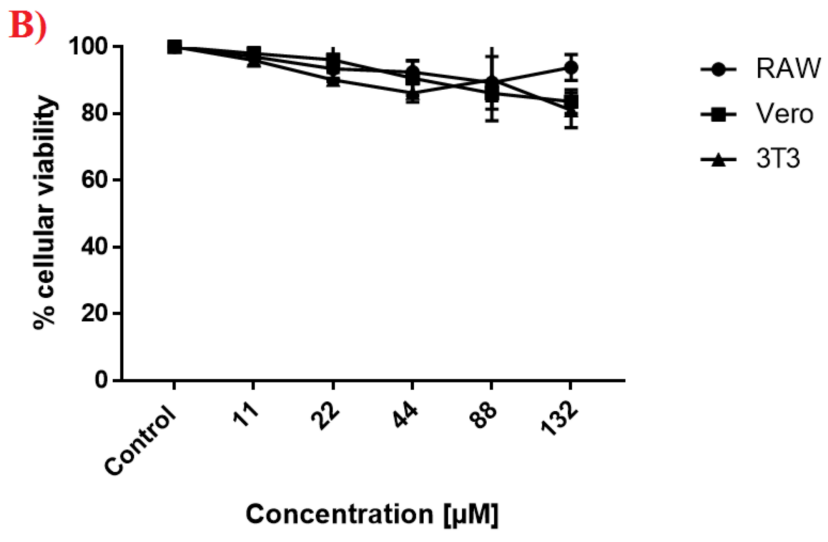

Figure 4. Viability of different cell lines $24 \mathrm{~h}$ after being exposed to different nanosystems. The cell lines RAW, 3T3, and VERO were exposed to different concentrations of DETC-loaded nanoparticles and drug-free nanoparticles for $24 \mathrm{~h}$. (A) DETC-loaded nanoparticles (NPD); (B) drug-free nanoparticles (NPB). Results presented as mean \pm standard deviation in an independent triplicate system and for the statistical analysis of the ANOVA test, together with Tukey's post hoc test; $\left.p<0.001{ }^{* * *}\right)$. In order to verify differences, the profile of each strain was compared against others treated with the same concentration of DETC nanoparticles and the controls.

\subsection{Capacity of Permeability Membrane Carrying Drugs}

To evaluate if nanoparticles with DETC could enter easily inside cells, we performed an assay to demonstrate this capacity. The results presented in Figure 5 demonstrate when nanoparticles were marked with FITC and put in contact for $24 \mathrm{~h}$ with two different cell lines (RAW and VERO). In the image is shown the presence of fluorescence inside cells after the process, and the cell line RAW demonstrates more intensity in fluorescence when compared with cell line VERO. 
RAW

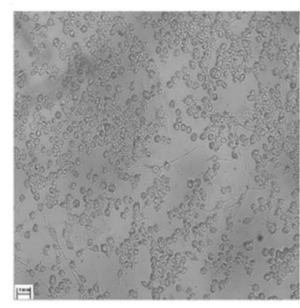

CONTROL

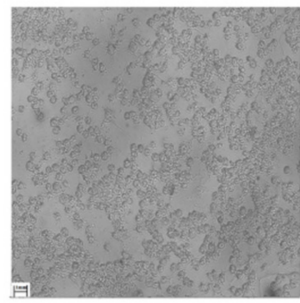

CONTROL

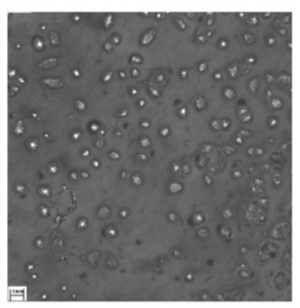

RAW - NP - L

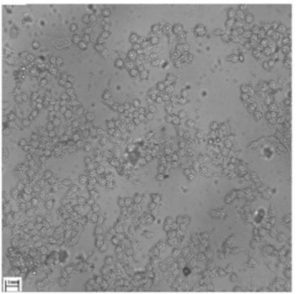

VERO - NP - L

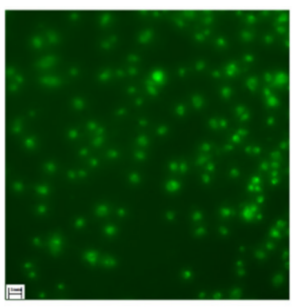

RAW - NP - F

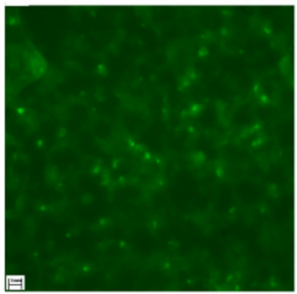

VERO - NP - F

Figure 5. Fluorescent microscopy of different cell lines $24 \mathrm{~h}$ after being exposed to stained DETCloaded nanoparticles. The cell lines RAW and VERO were exposed to DETC nanoparticles stained with FITC to evaluate if nanoparticles can enter inside cells, the scale bar is $0.1 \mathrm{~mm} / \mathrm{cm}$.

\subsection{Antiparasitic Activity against Trypanosoma cruzi}

The NPD antiparasitic activity was tested against different strains of T. cruzi in trypomastigote forms (Figure 6). The results show the inhibition curve of the parasite with an increase in the concentration of nanoparticles and how the different strains respond differently to the compound. The strains Dm28c and $\mathrm{Y}$ were demonstrated to be more susceptible to DETC nanoparticles when compared to the results with the strain Bolivia. In addition, we determined the $\mathrm{IC}_{50}$ based on the results for antiparasitic activity that can be observed in Table 2. In these results, beyond demonstrating the efficacy of the PLA-DETC nanoparticles, we demonstrate a simple comparison with the results of a commercial drug used against $T$. cruzi, benznidazole, whose IC $_{50}$ against these different strains is shown. It was possible to observe that DETC nanoparticles presented a lower $\mathrm{IC}_{50}$ than benznidazole.

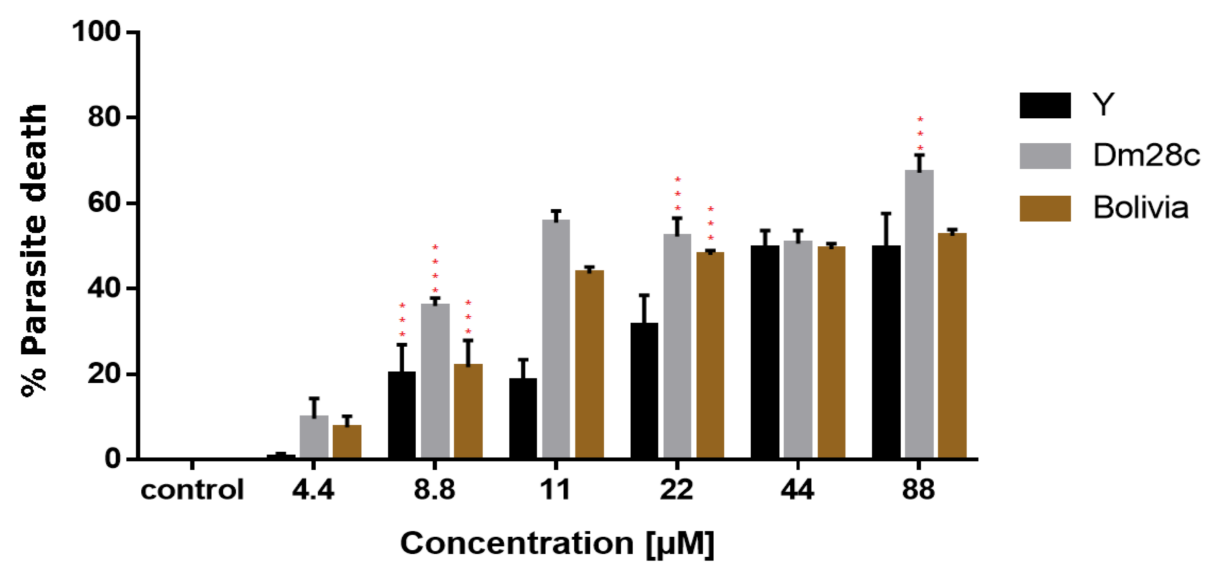

Figure 6. Trypanocidal activity of DETC nanoparticles against different strains of Trypanosoma cruzi. The parasites were exposed to different concentrations of DETC-loaded nanoparticles $(4.4 \mu \mathrm{M}$ to $88 \mu \mathrm{M}$ ) for $24 \mathrm{~h}$. Results are presented as mean \pm standard deviation of the percentage of parasitic inhibition in an independent triplicate system and for the statistical analysis of the ANOVA test, together with Tukey's post hoc test; $p<0.001\left(^{* * *}\right), p<0.0001\left(^{* * * *}\right)$. In order to verify differences, the profile of each strain was compared against others treated with the same concentration of DETC nanoparticles and the control. 
Table 2. IC $_{50}$ of DETC nanoparticles against different strains of T. cruzi after $24 \mathrm{~h}$ of exposure. Results are presented as mean \pm standard deviation in an independent triplicate system and for the statistical analysis of the ANOVA test, together with Tukey's post hoc test, in which different letters indicate statistically significant differences at $p<0.05$.

\begin{tabular}{ccc}
\hline \multirow{2}{*}{ Strain } & \multicolumn{2}{c}{ IC $_{\mathbf{5 0}}$ of Compounds against T. cruzi } \\
\cline { 2 - 3 } & NPD $(\boldsymbol{\mu M})$ & Benz $^{*}(\boldsymbol{\mu M})$ \\
\hline Dm28c & $15.47 \pm 2.71^{\mathrm{a}}$ & $70.58 \pm 6.87^{\mathrm{c}}$ \\
Y & $45.15 \pm 5.44^{\mathrm{b}}$ & $85.24 \pm 5.22^{\mathrm{d}}$ \\
Bolivia & $47.89 \pm 3.98^{\mathrm{b}}$ & $79.78 \pm 6.18^{\mathrm{c}}$ \\
\hline
\end{tabular}

${ }^{*}$ Note: Benz (benznidazole). a, b, c and d indicate statistical difference among groups.

\subsection{Stimulation of ROS Production by Trypanosoma cruzi after Exposure to DETC Nanoparticles}

To understand the mechanisms associated with DETC that cause the death of the parasite, the ROS production inside the parasite was analyzed and the results are shown in Figure 7. The nanoparticles of DETC interfere in ROS production by the parasites. In addition, the strains suffer differently against exposure to DETC. Strains Y and Dm28c were less affected by the nanoparticles than the strain Bolivia, which presented the highest ROS production. Furthermore, the parasites, when exposed to nanoparticles without DETC, demonstrated the capacity to increase ROS production.

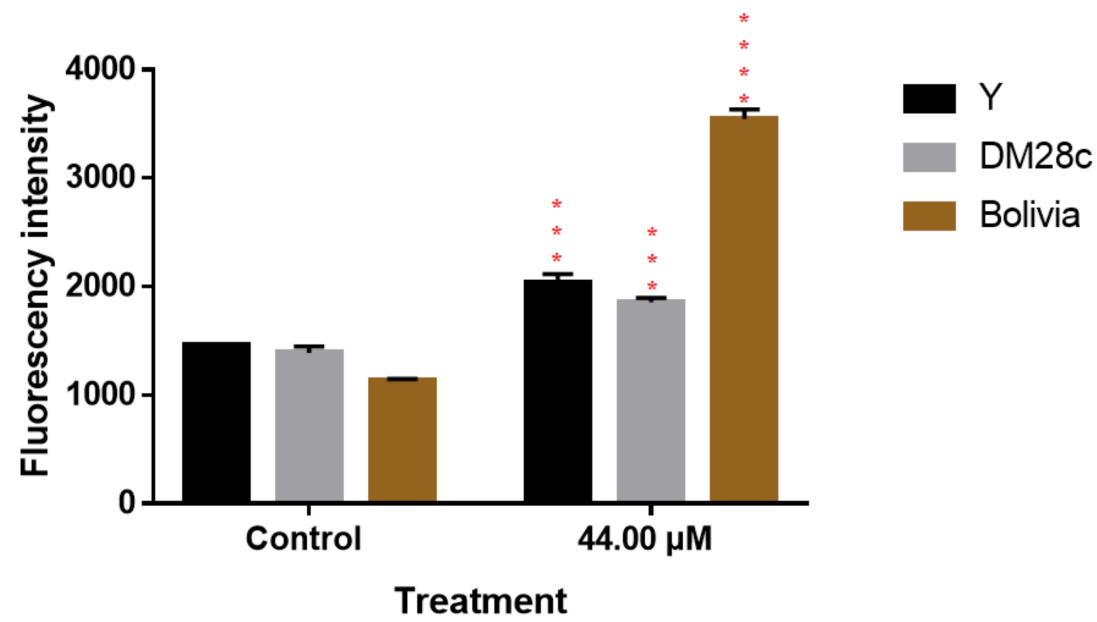

Figure 7. ROS production by Trypanosoma cruzi $24 \mathrm{~h}$ after being exposed to DETC nanoparticles. The parasites were exposed to a concentration of $44.0 \mu \mathrm{M}$ of NPD for $24 \mathrm{~h}$. Results are presented as mean \pm standard deviation of the percentage of parasitic inhibition in a triplicate system and for the statistical analysis of the ANOVA test, together with Tukey's post hoc test $\left(p<0.001\left(^{* * *}\right) ; p<0.0001\left(^{* * *}\right)\right.$. In order to verify differences, the profile of each strain was compared against the control.

\section{Discussion}

In this study, using biodegradable PLA nanoparticles as a promising nanotechnological approach to improve DETC activity was investigated. All formulations had small and monodispersible particle diameters. The average size being smaller than $200 \mathrm{~nm}(\mathrm{PdI}<0.3)$ demonstrates that the formulation led to the development of a narrow, uniform and singlemode particle size that is considered to be suitable to overcome biological barriers [29]. The distribution of nanoparticle diameters presents the following profile (Figure 1B), which confirms the formation of a single particle family (characterized by a unimodal particle distribution) located in the range between 100 and $200 \mathrm{~nm}$. The increase in particle size and the change in $\mathrm{pH}$ is indicative of DETC incorporation into NP, which is confirmed by the EE and DL measurements [30].

Luize Mazur (2018) revealed similar physicochemical results, showing a particle size of around $190 \mathrm{~nm}(\mathrm{PdI}<0.2)$ and negative zeta potential. However, the DETC encapsulation 
efficiency was close to $90 \%$ in solid lipid nanoparticles systems. Submicrometric sizes with a narrow distribution and satisfactory encapsulation efficiency are important in the development of nanosystems, especially for their biological application [31]. Furthermore, Assolini et al., in 2020, using double emulsion to develop a DETC-Beeswax-CO Nps using copaiba oil, obtained a nanoparticle with a size of $200 \mathrm{~nm}$. The system developed in this study presents a size of around $168 \mathrm{~nm}$, and the technique applied to encapsulation and the biopolymer used can be adapted to change the size of nanoparticles. In addition, the reduction in size could be an important point of treatment and lead to the elimination of parasites due to the capacity to enter cells and pierce their barriers [32].

In Table 3 below, we highlight some nanoparticles developed using DETC, a compound with different nanosystems, applied against the Tryponosomatidae family, specifically against Leishmania sp. However, in the parasitology field associated with nanoparticles, DETC was presented in a few studies for its application.

Table 3. Comparative table of findings in the literature on the use of nanoparticles with DETC against the Trypanosomatidae family.

\begin{tabular}{|c|c|c|c|c|c|}
\hline Name & Nanosystem & Size Obtained (nm) & Zeta $(\mathrm{mV})$ & $\begin{array}{c}\text { Efficacy } \\
\text { Encapsulation (\%) }\end{array}$ & Ref. \\
\hline DETC-Beeswax-copaiba & Double Emulsion & $\sim 190$ & $\sim-42$ & $\sim 90$ & [31] \\
\hline DETC-Beeswax-CO & Double Emulsion & $\sim 200$ & $\sim-44$ & $\sim 87$ & [32] \\
\hline PLA-DETC & Precipitation & $\sim 164$ & $\sim-20$ & $\sim 78$ & - \\
\hline
\end{tabular}

In order to investigate the interaction between the drug and the structural components, a spectroscopic analysis in the infrared region was performed. Comparing the spectra of physical mixtures in the 1:1 $(w / w)$ ratio of PLA/DETC with NPD showed a relevant difference. The physical mixture (PM) was prepared by using a PLA/DETC ratio of 1:1 w/w, which exhibited an FTIR spectrum similar to that recorded for the pure DETC. However, it is possible to observe important differences, such as the intensity of DETC bands and, significantly, the bathochromic shift of the ester band from PLA and the overlapping of the DECT bands. This phenomenon did not occur with drug-loaded nanoparticles, which corroborates the identification of a different drug-polymer interaction. Through comparisons of NPB vs. NPD in the FTIR spectrum, it is possible to identify a high intensity of the ester band at $1750 \mathrm{~cm}^{-1}$ in the drug-loaded nanoparticles [33].

The similarity of PM and free DETC can be associated with the hydrophobicity of DETC. When dissolved with PLA, this characteristic can be expressively elucidated by the results that we have obtained. In addition, we have clearly observed the reduction in the intensity of DETC peaks in the region of $2800-3000 \mathrm{~cm}^{-1}$ and around $800-1000 \mathrm{~cm}^{-1}$ when comparing PM and free DETC. These results are observed in other works using DETC, and the mixture is characterized by a reduction in the peaks of the compound $[31,32]$.

Cordeiro et al. (2021) showed the FTIR spectrum of the free DETC, and the free DETC spectra were similar to this study, with characteristic peaks at 837,909 , and $985 \mathrm{~cm}^{-1}$, representing the C-S bond, 1070 and 2104 for $C=S$, and also $1423 \mathrm{~cm}^{-1}$ for $\mathrm{N}-\mathrm{CS}_{2}$. Furthermore, DETC was co-encapsulated with 4-nitrohalcone in beeswax nanoparticles, and a greater intensity in the peaks of these compounds was observed according to the amount present in the formulation [25]. Furthermore, the same peaks in the FTIR were observed by Mazur et al. when developing nanoemulsions of DETC and beeswax, but they were less intense than our findings because they used a shorter concentration of DETC than in this experiment [31].

The experimental data show that DS and NPD exhibit different release behavior. In NPD, the DETC release rate was slower and more controlled compared to the pure drug solution. We suggest that the association with the polymeric shell causes a delay in the molecule dissemination. According to this, and associated to the adjustment in 
the parabolic kinetic model, it is implied that the release rate was controlled by diffusion dependent on the drug loading level [34].

DETC nanoparticles demonstrated low toxicity against different cell lines in Figure 4, and the results were similar to NPB. These results are associated with the controlled release of the drug and demonstrate the importance of nanoparticles for reducing toxicity, as observed in other studies, whereby the controlled release of drugs reduced the damage induction because of the minimal presence of the compound in cells. As observed in the results in Figure 3, the release of DETC was minimal when encapsulated, and that was essential to this result [35-39]. In addition, we observed that other studies using different nanosystems associated with DETC present a reduction in cellular toxicity compared with free DETC, and the results achieved by other researchers are similar at ours, with just a little toxicity when using elevated concentrations of nanoparticles with DETC [31,32].

In addition, associated with the test of cytotoxicity, we performed an assay using stained NPD with FITC (Figure 5). We observed that nanoparticles can enter the cells, and this is an important point when validating tests against Chagas disease in the chronic stage, principally when the objective is the production of new drugs using new technologies is to improve the efficacy and safety of organisms [40,41].

The nanosystem proprieties that we produced also demonstrate its capacity to enter into cells, since the PLA coating presents characteristics that facilitate association with membranes $[42,43]$. These characteristics are possible due to the polarization of PLA nanoparticles, or polymeric nanoparticles, aiding their attachment to membranes and helping to pierce through them. Furthermore, PLA is a component approved by the FDA, and its presence in the literature as a material against T. cruzi is positive, with good prospects regarding its ability to improve efficacy and safety [14,44]. Furthermore, some studies have demonstrated the capacity of PLA nanoparticles to penetrate the cellular membrane and barriers [45-47].

Moreover, DETC nanoparticles present a high antiparasitic activity, as shown in Figure 6. PLA-DETC nanoparticles were tested against different strains of T. cruzi (Dm28c, $\mathrm{Y}$, and Bolivia), and these strains present variable genetic patterns, evidencing that DETC nanoparticles can affect different variants of the genome of the parasite. The genetic variation resulted in different profiles of parasite death during the experiment after exposure to the compound, and this variation is normal because the alteration in genetic patterns can result in alterations in resistance [48,49].

Furthermore, when comparing the results achieved here with free DETC, we observed a similar profile of $\mathrm{IC}_{50}$ for free DETC and PLA-DETC nanoparticles against T. cruzi. These results indicate that the encapsulation of DETC did not reduce the capacity to eliminate the parasite, although there was a controlled release of drug by the nanosystem [9].

We found that the parasite, when exposed to PLA-DETC, increased ROS production (as visualized in Figure 7). This is due to the DETC present in an amine group, which acts in ROS production, and this intensifies the ROS production inside the parasite. The accumulation of reactive species induces damage to the parasite and its posterior death [7-9]. The literature already demonstrates the capacity of DETC to increase ROS production in its free form when the parasite is exposed to it. Furthermore, the literature demonstrated the importance of ROS production to the death of the parasite via the accumulation of damage caused by molecular oxidation [6-9,32].

Recently, a study using disulfiram, in which DETC is produced metabolically during degradation, demonstrated its promise in the treatment of T. cruzi, and demonstrated high activity, showing that it was capable, when associated with BNZ, to lead a better result against the parasite [50]. These findings demonstrate the importance of using the metabolic compound of disulfiram in new tests and mechanisms of delivery, which would also increase the probability of the compound DETC, when associated with nanoparticles, being used as a new drug for the treatment of Chagas disease.

This study demonstrates a possible new drug that could be used in CD treatment, presenting results that demonstrate the great potential of DETC nanoparticles, which are 
associated with viability for cells and high levels of damage to parasites. However, in vivo studies in preclinical models will be necessary to enhance the information presented in this work, such as toxicological profile analysis, antiparasitic activity analysis, and pharmacokinetic profiling, which are the important next steps of this study.

Furthermore, CD is a disease that principally affects poor regions of the world, making necessary the use of systems, models, and technologies with low cost to guarantee that a newly discovered drug can help the regions most affected by the disease. In this study, the product developed, PLA-DETC nanoparticles, can alleviate these concerns. The technology and the methods in this study involve low costs when fabricating the PLA-DETC nanosystem. In addition, both main compounds are cheap to acquire, and the production system used was easy and fast.

\section{Conclusions}

The PLA-DETC nanosystem presents high stability and a small size of around $\sim 164 \mathrm{~nm}$, which was verified by SEM, AFM, and DLS analyzes. The PLA-DETC nanoparticles demonstrate controlled drug release, which was proven in an in vitro study. Moreover, that capacity is associated with the low toxicity of the system against three cell lines RAW, 3T3, and VERO, which was verified in vitro, demonstrating that the nanoparticles are safe and able to reduce DETC toxicity. In addition, the capacity of nanoparticles to enter the cells was proven by fluorescence, using nanoparticles stained with FITC. This is an important mechanism for the treatment of the chronic stage of CD due to the increase in the bioavailability of the compound. In addition, PLA-DETC presented high antiparasitic activity against different strains ( $\mathrm{Y}, \mathrm{Dm} 28 \mathrm{c}$, and Bolivia) of T. cruzi in trypomastigote forms, and also caused an increase in the ROS production of the parasites, showing that exposure to the system can cause damage and posterior death. The results demonstrated the importance of the PLA-DETC nanosystem as a possible alternative to the treatment of Chagas disease, especially for its use in the chronic stage of the disease, which does not have an efficient treatment.

Author Contributions: Experimental study design: J.W.d.F.O., M.F.A.d.S., A.A.d.S.J., H.A.O.R. and M.S.S.; Cultivation of parasites and assessment of antiparasitic activity: J.W.d.F.O. and M.S.S.; Scanning electron microscopy methodology and data analysis: J.W.d.F.O. and I.Z.D.; Obtaining research funding: H.A.O.R., A.A.d.S.J. and M.S.S. In addition, all authors actively participated in the writing and discussion of the manuscript. All authors have read and agreed to the published version of the manuscript.

Funding: This research was funded by Global Health and Tropical Medicine: Grant number IHMTUID/multi/04413/2013 and Grant number PTDC/CVT-CVT/28908/2017, FCT-Portugal.

Acknowledgments: We would like to thank João Aristeu da Rosa and Aline Rimodi Rimeiro at UNESP (Universidade Estadual de São Paulo) Araraquara (Brazil) for offering four different strains of T. cruzi. JWFO and MFAS give thanks to their financial support (PhD and post-doctoral fellowships) provided by Capes/Brazil; MSS, AASJ, and HAOR give thanks to CNPq/Brazil for the Research Grant (Bolsa de Produtividade). We would also like to thank the Department of Materials Engineering at UFRN for affording us the use of their scanning electron microscope, and the Department of Biochemistry at UFRN for affording us the use of their culture room. We thank Laysa Ohana for reviewing and editing the English version of the manuscript.

Conflicts of Interest: The authors declare no conflict of interest.

\section{References}

1. Navarro, M.; Monge-Maíllo, B.; Flores-Chavez, M.D.; López-Vélez, R. Hunting hidden parasites: Trypanosoma cruzi. Lancet 2017, 390, 724-726. [CrossRef]

2. Castillo-Riquelme, M. Chagas disease in non-endemic countries. Lancet Glob. Health 2017, 5, e379-e380. [CrossRef]

3. Marín, P.A.; Soto-Ospina, A. Redox mechanism of Trypanosoma cruzi resistance to nitro prodrugs Benznidazole and Nifurtimox. Int. J. Bioinforma. Comput. Biol. 2020, 5, 1-7. 
4. Buschini, A.; Ferrarini, L.; Franzoni, S.; Galati, S.; Lazzaretti, M.; Mussi, F.; de Albuquerque, C.N.; Zucchi, T.M.A.D.; Poli, P. Genotoxicity revaluation of three commercial nitroheterocyclic drugs: Nifurtimox, benznidazole, and metronidazole. J. Parasitol. Res. 2009, 2009. [CrossRef]

5. Urbina, J.A. Recent clinical trials for the etiological treatment of chronic Chagas disease: Advances, challenges and perspectives. J. Eukaryot. Microbiol. 2015, 62, 149-156. [CrossRef]

6. Oliveira, J.W.D.; Rocha, H.A.O.; de Medeiros, W.M.T.Q.; Silva, M.S. Application of Dithiocarbamates as Potential New Antitrypanosomatids-Drugs: Approach Chemistry, Functional and Biological. Molecules 2019, 24, 2806. [CrossRef]

7. Khouri, R.; Novais, F.; Santana, G.; de Oliveira, C.I.; Santos, M.A.V.D.; Barral, A.; Barral-Netto, M.; van Weyenbergh, J. DETC induces Leishmania parasite killing in human in vitro and murine in vivo models: A promising therapeutic alternative in leishmaniasis. PLoS ONE 2010, 5, e14394. [CrossRef]

8. Celes, F.S.; Trovatti, E.; Khouri, R.; van Weyenbergh, J.; Ribeiro, S.J.L.; Borges, V.M.; Barud, H.S.; de Oliveira, C.I. DETC-based bacterial cellulose bio-curatives for topical treatment of cutaneous leishmaniasis. Sci. Rep. 2016, 6, 1-11. [CrossRef]

9. Oliveira, J.W.d.; Torres, T.M.; Moreno, C.J.G.; Amorim-Carmo, B.; Damasceno, I.Z.; Soares, A.K.M.C.; Barbosa, J.D.; Rocha, H.A.O.; Silva, M.S. Insights of antiparasitic activity of sodium diethyldithiocarbamate against different strains of Trypanosoma cruzi. Sci. Rep. 2021, 11, 1-13.

10. Ferraz, L.R.M.; Silva, L.C.P.B.B.; de Souza, M.L.; Alves, L.P.; Sales, V.D.W.; Barbosa, I.D.N.G.; de Andrade, M.C.; Santos, W.M.D.; Rolim, L.A.; Rolim-Neto, P.J. Drug associations as alternative and complementary therapy for neglected tropical diseases. Acta Trop. 2022, 225, 106210. [CrossRef]

11. Parvez, S.; Yadagiri, G.; Gedda, M.R.; Singh, A.; Singh, O.P.; Verma, A.; Sundar, S.; Mudavath, S.L. Modified solid lipid nanoparticles encapsulated with Amphotericin B and Paromomycin: An effective oral combination against experimental murine visceral leishmaniasis. Sci. Rep. 2020, 10, 1-14. [CrossRef] [PubMed]

12. Kimani, N.M.; Backhaus, S.; Matasyoh, J.C.; Kaiser, M.; Herrmann, F.C.; Schmidt, T.J.; Langer, K. Preparation of Sesquiterpene LactoneLoaded PLA Nanoparticles and Evaluation of Their Antitrypanosomal Activity. Molecules 2019, 24, 2110. [CrossRef] [PubMed]

13. Branquinho, R.T.; de Mello, C.G.; Oliveira, M.T.; Reis, L.E.; de Abreu Vieira, P.M.; Mosqueira, V.C.; de Lana, M. Lychnopholide in PLA-PEG nanocapsules cures infection by drug resistant Trypanosoma cruzi strain in acute and chronic phases. Antim. Agents Chemo. 2020, 64, 4 .

14. Donsì, F.; Ferrari, G. Changing the Vision in Smart Food Design Utilizing the Next Generation of Nanometric Delivery Systems for Bioactive Compounds. Foods 2020, 9, 1100. [CrossRef]

15. dos-Santos-Silva, E.; Alves-Silva, M.F.; de Medeiros, J.S.; Santos-Cavalcante, R.D.; Cornélio, A.M.; Fernandes-Pedrosa, M.F.; Egito, E.S.T.D.; de Araújo-Júnior, R.F.; da Silva-Júnior, A.A. Colloidal properties of self-assembled cationic hyperbranchedpolyethyleneimine covered poly lactide-co-glycolide nanoparticles: Exploring modified release and cell delivery of methotrexate. J. Mol. Liq. 2020, 315, 113721. [CrossRef]

16. Lima, T.L.C.; Feitosa, R.D.; Santos-Silva, D.; Santos-Silva, D.; Maria, A.; Siqueira, E.M.D.; Machado, P.R.L.; Cornélio, A.M.; Egito, E.S.T.D.; Fernandes-Pedrosa, M.D. Improving encapsulation of hydrophilic chloroquine diphosphate into bio degradable nanoparticles: A promising approach against herpes virus simplex-1 infection. Pharmaceutics 2018, 10, 255. [CrossRef]

17. Zhao, J.; Stenzel, M.H. Entry of Nanoparticles into Cells: The Importance of Nanoparticle Properties. Polym. Chem. 2018, 9, 259-272. [CrossRef]

18. Breunig, M.; Bauer, S.; Göpferich, A. Polymers and Nanoparticles: Intelligent Tools for Intracellular Targeting? Europ. J. Pharm. Biopharm. 2008, 68, 112-128. [CrossRef]

19. Hadji, H.; Bouchemal, K. Effect of micro- and nanoparticle shape on biological processes. J. Control Release 2021, 342, 93-110. [CrossRef]

20. Contreras, V.T.; Salles, J.M.; Thomas, N.; Morel, C.M.; Goldenberg, S. In vitro differentiation of Trypanosoma cruzi under chemically defined conditions. Mol. Biochem. Parasitol. 1985, 16, 315-327. [CrossRef]

21. Mosmann, T. Rapid colorimetric assay for cellular growth and survival: Application to proliferation and cytotoxicity as says. J. Immunol. Methods 1983, 65, 55-63. [CrossRef]

22. de Oliveira, A.R.; Mesquita, P.C.; Machado, P.R.L.; Farias, K.J.S.; de Almeida, Y.M.B.; Fernandes-Pedrosa, M.F.; Cornélio, A.M.; Egito, E.S.T.D.; da Silva-Júnior, A.A. Monitoring structural features, biocompatibility and biological efficacy of gamma-irradiated methotrexate-loaded spray-dried microparticles. Mater. Sci. Eng. C 2017, 80, 438-448. [CrossRef] [PubMed]

23. Mohammadi, I.; Shahrabi, T.; Mahdavian, M.; Izadi, M. Chemical modification of LDH conversion coating with diethyldithiocarbamate as a novel anti-corrosive film for AA2024-T3. J. Ind. Eng. Chem. 2021, 95, 134-147. [CrossRef]

24. Hickey, J.W.; Santos, J.L.; Williford, J.-M.; Mao, H.-Q. Control of polymeric nanoparticle size to improve therapeutic delivery. J. Control Release 2015, 219, 536-547. [CrossRef]

25. Cordeiro, A.P.; Feuser, P.E.; Figueiredo, P.G.; da Cunha, E.S.; Martinez, G.R.; Machado-de-Ávila, R.A.; Rocha, M.E.M.; de Araújo, P.H.H.; Sayer, C. In vitro synergic activity of diethyldithiocarbamate and 4-nitrochalcone loaded in beeswax nanoparticles against melanoma (B16F10) cells. Mater. Sci. Eng. C 2021, 20, 111651. [CrossRef]

26. Qi, F.; Zhang, X.; Li, S. A novel method to get methotrexatum/layered double hydroxides intercalation compounds and their release properties. J. Phys. Chem. Solids. 2013, 74, 1101-1108. [CrossRef] 
27. Nascimento, E.G.D.; de Azevedo, E.P.; Alves-Silva, M.F.; Aragão, C.F.S.; Fernandes-Pedrosa, M.F.; da Silva-Junior, A.A. Supramolecular aggregates of cyclodextrins with co-solvent modulate drug dispersion and release behavior of poorly soluble corticosteroid from chitosan membranes. Carbohydr. Polym. 2020, 248, 116724. [CrossRef]

28. da Costa, P.J.C. Avaliação in vitro da lioequivalência de formulações farmacêuticas. Rev. Bras. Ciências Farm. 2002, 38, 141-153. [CrossRef]

29. Juère, E.; Kleitz, F. On the nanopore confinement of therapeutic drugs into mesoporous silica materials and its implications. Microporous Mesoporous Mater. 2018, 270, 109-119. [CrossRef]

30. Silva, A.M.D.S.; de Caland, L.B.; Doro, P.N.D.; Oliveira, A.L.C.D.L.; de Araújo-Júnior, R.F.; Fernandes-Pedrosa, M.F.; Egito, E.S.T.D.; da Silva-Junior, A.A. Hydrophilic and hydrophobic polymeric benznidazole-loaded nanoparticles: Physicochemical properties and in vitro antitumor efficacy. J. Drug Deliv. Sci. Technol. 2019, 51, 700-707. [CrossRef]

31. Mazur, K.L.; Feuser, P.E.; Valério, A.; Cordeiro, A.P.; de Oliveira, C.I.; Assolini, J.P.; Pavanelli, W.R.; Sayer, C.; Araújo, P.H.H Diethyldithiocarbamate loaded in beeswax-copaiba oil nanoparticles obtained by solventless double emulsion technique promote promastigote death in vitro. Colloids Surf. B Biointerfaces 2019, 176, 507-512. [CrossRef]

32. Assolini, J.P.; Tomiotto-Pellissier, F.; da Silva Bortoleti, B.T.; Goncalves, M.D.; Sahd, C.S.; Carloto, A.C.M.; Feuser, P.E.; Cordeiro, A.P.; Borghi, S.M.; Verri, W.A., Jr. Diethyldithiocarbamate Encapsulation Reduces Toxicity and Promotes Leishmanicidal Effect through Apoptosis-like Mechanism in Promastigote and ROS Production by Macrophage. J. Drug Target. 2020, 28, 1110-1123. [CrossRef]

33. Dendisová, M.; Jeništová, A.; Parchaňská-Kokaislová, A.; Matějka, P.; Prokopec, V.; Švecová, M. The use of infrared spectroscopic techniques to characterize nanomaterials and nanostructures: A review. Anal. Chim. Acta 2018, 1031, 1-14. [CrossRef]

34. Santos-Silva, A.M.D.; de Caland, L.B.; Oliveira, A.L.C.D.L.; de Araújo-Júnior, R.F.; Fernandes-Pedrosa, M.F.; Cornélio, A.M.; da Silva-Júnior, A.A. Designing structural features of novel benznidazole-loaded cationic nanoparticles for inducing slow drug release and improvement of biological efficacy. Mater. Sci. Eng. C 2017, 78, 978-987. [CrossRef]

35. Ajdary, M.; Moosavi, M.A.; Rahmati, M.; Falahati, M.; Mahboubi, M.; Mandegary, A.; Jangjoo, S.; Mohammadinejad, R.; Varma, R.S. Health concerns of various nanoparticles: A review of their in vitro and in vivo toxicity. Nanomaterials 2018, 8, 634. [CrossRef]

36. Ray, B.; Bisht, S.; Maitra, A.; Maitra, A.; Lahiri, D.K. Neuroprotective and neurorescue effects of a novel polymeric nanoparticle formulation of curcumin (NanoCurc ${ }^{\mathrm{TM}}$ ) in the neuronal cell culture and animal model: Implications for Alzheimer's disease. J. Alzheimer's Dis. 2011, 23, 61-77. [CrossRef]

37. Nishihira, V.S.K.; Fontana, B.D.; Ianiski, F.R.; de Almeida, H.S.; Posser, C.P.; Dias, J.B.; Parodi, C.B.; Piva, M.M.; Gris, A.; Mendes, R.E. PEGylated meloxicam-loaded nanocapsules reverse in vitro damage on caspase activity and do not induce toxicity in cultured human lymphocytes and mice. Biomed. Pharmacother. 2018, 107, 1259-1267. [CrossRef]

38. Chen, W.; Li, D.; Ahmed, E.-S.; El-Newehy, M.; Ei-Hamshary, H.A.; Al-Deyab, S.S.; He, C.; Mo, X. Dexamethasone loaded coreshell SF/PEO nanofibers via green electrospinning reduced endothelial cells inflammatory damage. Colloids Surf. B Biointerfaces 2015, 126, 561-568. [CrossRef]

39. Kamaly, N.; Yameen, B.; Wu, J.; Farokhzad, O.C. Degradable controlled-release polymers and polymeric nanoparticles: Mechanisms of controlling drug release. Chem. Rev. 2016, 116, 2602-2663. [CrossRef]

40. Arrúa, E.C.; Seremeta, K.P.; Bedogni, G.R.; Okulik, N.B.; Salomon, C.J. Nanocarriers for effective delivery of benznidazole and nifurtimox in the treatment of chagas disease: A review. Acta Trop. 2019, 198, 105080. [CrossRef]

41. Salomon, C.J. First century of Chagas' disease: An overview on novel approaches to nifurtimox and benznidazole delivery systems. J. Pharm. Sci. 2012, 101, 888-894. [CrossRef]

42. Raj, S.; Khurana, S.; Choudhari, R.; Kesari, K.K.; Kamal, M.A.; Garg, N.; Ruokolainen, J.; Das, B.C.; Kumar, D. Specific targeting cancer cells with nanoparticles and drug delivery in cancer therapy. In Seminars in Cancer Biology; Elsevier: Amsterdam, The Netherlands, 2021; pp. 166-177.

43. Casalini, T.; Rossi, F.; Castrovinci, A.; Perale, G. A perspective on polylactic acid-based polymers use for nanoparticles synthesis and applications. Front. Bioeng. Biotechnol. 2019, 7, 259. [CrossRef]

44. Goudarzi, F.; Asadi, A.; Afsharpour, M.; Jamadi, R.H. In Vitro Characterization and Evaluation of the Cytotoxicity Effects of Nisin and Nisin-Loaded PLA-PEG-PLA Nanoparticles on Gastrointestinal (AGS and KYSE-30), Hepatic (HepG2) and Blood (K562) Cancer Cell Lines. AAPS Pharm. Sci. Tech. 2018, 19, 1554-1566. [CrossRef]

45. Selby, L.I.; Cortez-Jugo, C.M.; Such, G.K.; Johnston, A.P. Nanoescapology: Progress toward Understanding the Endosomal Escape of Polymeric Nanoparticles. Wiley Interd. Rev. Nanom. Nanobiotech. 2017, 9, e1452. [CrossRef]

46. Khalid, M.; El-Sawy, H.S. Polymeric Nanoparticles: Promising Platform for Drug Delivery. Int. J. Pharm. 2017, 528, 675-691.

47. Zhang, T.-T.; Li, W.; Meng, G.; Wang, P.; Liao, W. Strategies for Transporting Nanoparticles across the Blood-Brain Barrier. Biomat. Sci. 2016, 4, 219-229. [CrossRef]

48. Jimenez, V. Dealing with environmental challenges: Mechanisms of adaptation in Trypanosoma cruzi. Res. Microbiol. 2014, 165, 155-165. [CrossRef]

49. Zingales, B. Trypanosoma cruzi genetic diversity: Something new for something known about Chagas disease manifestations, serodiagnosis and drug sensitivity. Acta Trop. 2018, 184, 38-52. [CrossRef]

50. Saraiva, R.M.; Portela, L.F.; da Silveira, G.P.; da Silva Gomes, N.L.; Pinto, D.P.; da Silva, A.C.; Sangenis, L.H.; Carneiro, F.M.; Almeida-Silva, J.; Marinho, P.W.; et al. Disulfiram Repurposing in the Combined Chemotherapy of Chagas Disease: A Protocol for Phase I/II Clinical Trial. Med. Case Rep. Study Prot. 2021, 2, e0110. [CrossRef] 